Article

\title{
Moving Spaces: Mapping the Drama Room as Heterotopia
}

\section{Elsa Szatek}

Department of Humanities and Social Sciences Education [HSD], Stockholm University, Stockholm 10691, Sweden; elsa.szatek@hsd.su.se

Received: 5 February 2020; Accepted: 5 March 2020; Published: 10 March 2020

\begin{abstract}
This article is aimed at exploring the political characteristics of the drama space, which reflects, juxtaposes, and opposes particular sites in a participant's everyday life, such as the school. By putting spatial theories to work, this article investigates the drama space belonging to an all-girls community group in Sweden, participation in which is voluntary and where the artistic work produced relies on a democratic process, with the girls' input being vital. I conceptualise the drama room as a heterotopia that functions as an exclusive and excluding space as a well as a space of resistance. Based on interviews with the girls, this ethnographic study challenges the conventional notion that applied drama is only an interrelational matter between the drama participants. By examining the drama room's role as the 'other place' in the girls' everyday lives while being connected to 'everyday' places, this article demonstrates the drama room as an important space for the girls to have agency, there and elsewhere. When placing space and place in the foreground, a 'dramaspaceknowledge' emerges, the influence of which stretches beyond the drama room. This article argues that the girls' dramaspaceknowledge is utilised when creating a performance and while challenging structures and norms elsewhere, such as in their schools and communities.
\end{abstract}

Keywords: community theatre; applied drama; heterotopia; supplementary education; public school 


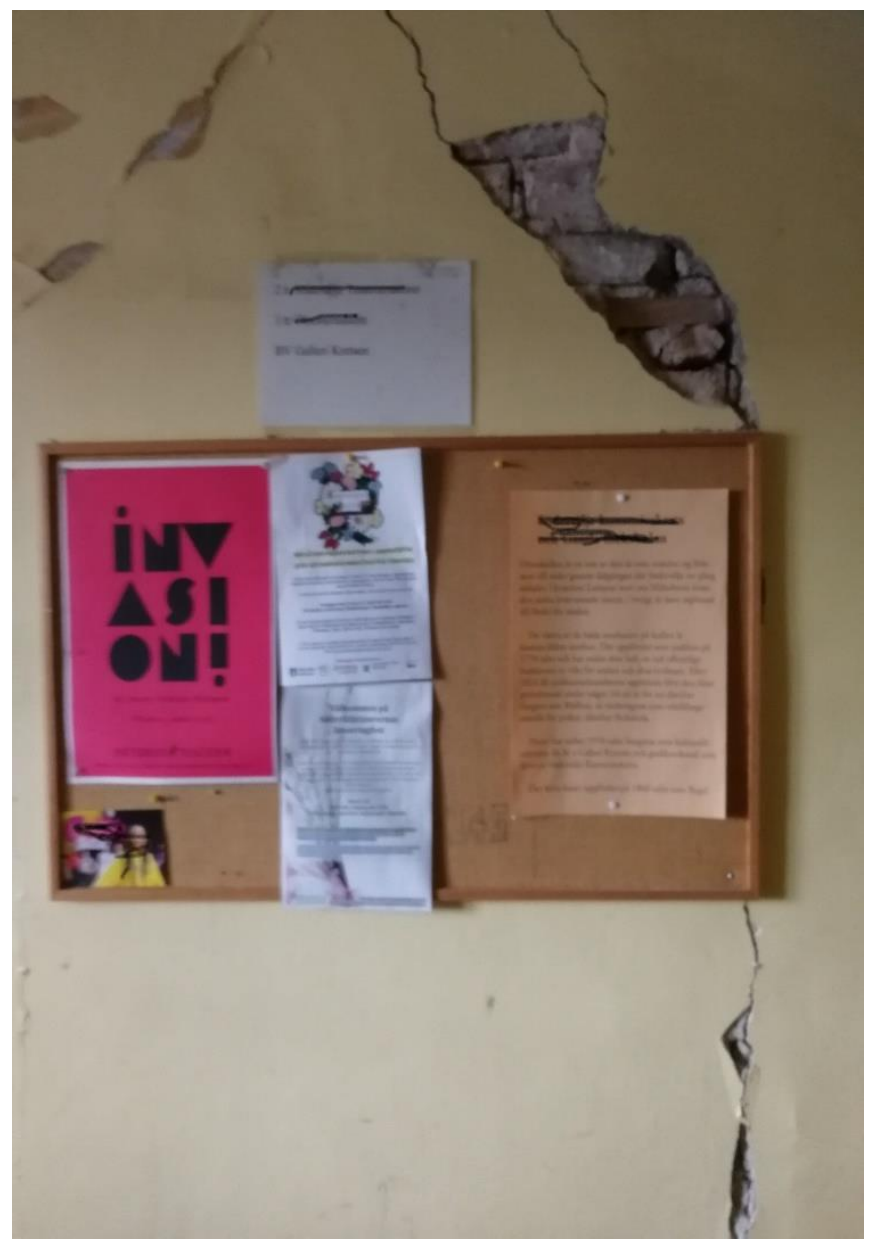

Sweden, Spring 2019: I am climbing the stairs in a dilapidated building from the beginning of the last century which houses an all-girls community theatre group. The walls in the hallway are cracked, exposing the mortar behind the facade and my steps are echoing against the rundown marble steps. I'm surrounded by the smell that I've come to connect to this particular place; a mixture of old dust from the crumbled walls and fresh air squiggling in through the window frames. As I leave the staircase behind I'm entering a large, open room with wooden floors and large windows offering a stunning view of the town and the landscape beyond. It is evening, in the middle of the week and 15 girls aged $13-17$ have made their way from their different schools and are now gathered in a circle on the floor, some of them sitting, others kneeling or lying down. The girls meet here once a week to explore issues related to their everyday life as they turn into young women, share experiences, visions and dreams. There is an equal mix of vivid discussions and embodied practice, often the two are entangled and the borders between reality and fiction are frequently pushed, erased and blurred. From one of the windows I have a splendid view of the town which houses about 75000 citizens, many of them with a background other than Swedish. From where I'm standing I overlook industries that employs many of the citizens in the town and high-rise buildings that houses many of those working there. And far, away among hills covered in trees, I spot some villas. This evening there is a lively discussion of their perception of what it's like to live and grow up in their particular urban space in Sweden. Tove the leader, is trying her best to make sure everybody can have their say, which is easier said than done, as there are many points of view in the group. 'This town is more conservative than Syria where I fled from two years ago' exclaims Amina. She is talking about honour violence that many of the girls in this group have direct or indirect experience of. Sarah, on the other hand, feels safe most of the time when in school although she recognizes that the town is segregated. Alya tells a story of boys being 
dominant in her school setting the agenda of what to say and how to behave. The discussions go back and forth for almost an hour before Tove, finally breaks up the circle and encourages the girls to put their talk into scenes. From her perspective important matters have been put into words, issues she thinks are vital for the performance the group are in the process of devising.

\section{Points of Departure}

The participants above represent some of the 20,000 or so adolescent girls who practise drama as a spare time activity in Sweden, where theatre does not appear in the school curriculum until high school, and even then it is an optional activity. Those who participate are usually interested in drama and theatre, with some even planning to join an amateur theatre club or attend an arts school. Embodying different roles in fictional contexts and exploring and expressing issues of concern make for an affective experience for many of the girls. The explorative drama work takes place in a closed drama room and might lead up to a performance that is staged in front of an audience. Researchers within the fields of applied drama and theatre note that participants in theatrical activities gain skills such as control over one's body and voice, co-creating with others, and learning to improvise in inventive ways [1-3], as shown by the results of studies of drama and theatre groups that are particularly for girls [4-8]. Many of these skills are also linked to being a democratic citizen, the reason for which drama and theatre tend to appear in activities such as conflict resolution and are often featured in projects exploring the discussion of social issues such as bullying, conflict management, and citizenship $[1,2,9,10]$. The results of the studies showed that drama and theatre have many positive effects, such as increased self-esteem, creativity, agency, and empathy, in addition to gaining the artistic skills that are required for theatre practice. However, I also see the need to explore the manner in which this knowledge works in a transgressive way between the drama room and the everyday lives of the girls. To address this issue, my focus in this article is on contextualising the space in which drama takes place.

The kind of drama that is explored in this article would best be labelled as community theatre. In this context, the community is defined as a 'virtue of a shared primary identity based in place, ethnicity, class, race, sexual preference, profession, circumstances, or political orientation' [11] (p. 2). The community in this case study consists of girls, turning into young women, striving to challenge and explore norms imposed on the female body in this particular town. Community, together with theatre, indicates a group of people creating theatre in relation to a certain place or sense of identity. Alternatively, it can refer to theatre emerging from a specific place or concern because it has a story that needs telling. A theatre emerging from a place or concern is rarely a scripted play. Rather, the theme and the text evolves in a rhizomatic process from a group in a specific place. In my case it is young girls living in a certain urban place where many find themselves limited by a culture of chastity and control. One of the girls in the theatre group, Sarah, likens it to always being watched: 'they will keep their eyes on you no matter what, they know what happens. It is like people around you know what happens, one can never hide... I can't be myself' [Interview February, 2020]. As a response to being limited by male norms, the theatre group provides a space where feministic issues are explored and expressed through artistic practice that emerges from the girls' expression and stories. Community theatre is a response to the everyday, always entangled with place and those living there, it is a theatre which values the relationship it has with people's everyday lives' [12] (p. 11). The everyday practice is understood to be:

habitual but not unchangeable and is therefore worth taking seriously, for it is the reality which we are made aware of when theatre is good and return to when the theatre is done. [12] (p. 17)

The drama room-from which the theatre emerges before it is shown to an audience-is a room entangled with everyday life. It is important for my study to understand this entanglement-how the town and its every day places creep in through the walls of the drama room affecting the space and the doings in that space. Simultaneously, the knowledge produced in the drama room leaks out, entangling itself with the school, the town, and other places of knowledge production. Thus, the drama 
room is not an isolated place. Rather, it has 'cracks in the walls'. This article follows the movements leaking through these cracks.

Underlying my research are spatial theories derived from Michel Foucault and Doreen Massey, informed by the idea of a drama space as a process, constantly in becoming. The social nature of its productionenables multiple stories and trajectories [13]. Trajectories are here interpreted as irreversible processes producing change [in line with Massey], rather than a movement that eventually flattens out. These trajectories and stories emerge from the relations in a space where they can be interpreted as 'embedded practices' [13] (p. 10). Considering space as a process means that space is always an open system rather than a static entity that is constructed not only by the relations there but by politics, culture, and economics. Space is thus always entangled with the everyday life and what is inside or outside becomes a rather complex process $[13,14]$. This entanglement is interesting to explore by using heterotopia as a concept. When interpreting the drama room as a heterotopia, the processes of becoming in that space are understood to be both separated from and entangled with local culture, history, and norms [15]. One purpose of exploring the drama room as a heterotopia is to explore and identify the everyday places that are being inverted, juxtaposed, and reflected there. The construction of space is a transversal process rather than being understood as a dualistic approach wherein different places and spaces are interpreted to be separate. Comprehending the intertwined relationship between the drama room and the girls' schools is important to efforts directed towards democracy and citizenship with adolescent girls because much of their time is spent in school. By investigating the entanglement between the drama room, the school, and the town in which the girls live, I wish to highlight the issues that may arise when drama with emancipatory ambitions is put to work. In this drama space, artistic practice and social ambition are entangled. There is also an emancipatory dimension as tacit knowledge from the everyday is made explicit.

This article maps the transversal relation between the production of space, place, and knowledge to explore ways in which activities in the drama space relate to other places such as school and the town. My ambition is to disentangle the threads that constitutes the relationship between applied theatre and citizenship, some which has been taken for granted. As part of a wider study, this article is focused on two questions: How is the drama space co-constructed in relation with other places in the girls' lives? What knowledges are articulated by the girls in relation to the drama room and other places?

\section{Materials and Methods}

\subsection{The Drama Room As a Productive Space}

Positioning myself somewhere between post-structuralism and posthuman philosophy, I recognize that space and materialism are more than just a backdrop to human agency. In line with Rosie Braidotti $[16,17]$ the subject is considered to be relational with multiple belongings and always embedded in context. The human subject is thus understood to constantly be in a transversal relation with place, space, other beings and materiality. There is no constant self, nor an ideal destination at which to arrive; thus, the human subject is nomadic in nature. Gilles Deleuze and Felix Guattari [18] [p. 1] put it beautifully, writing that 'each of us were several' suggesting than one person holds multiple belongings and personalities. As a drama researcher I recognize the manner in which this idea relates to agency, creativity, and imagination.

In this article, the drama room is encountered as a physical place, holding a multiplicity of trajectories, stories, and experiences in line with Massey's theories of place [13]. The relations that occur in the drama room produce a socially constructed space that enables a multiplicity of stories to co-exist. Conceived thus, just as a place, it is always under construction. It is because of this process of becoming that space could be 'imagined as a simultaneity of stories so far' [13] (p. 9). Space is constructed in a transversal process with place and social interaction. The physical place affects what social spaces are possible to create and the social practices produced in a space will change the 
meaning of the physical place. With regard to posthumanism [Karen Barad in particular] and new materialism, space, knowledge, and power can be understood to be entangled and unrepeatable in a 'spaceknowledgepower' formation, indicating that they are mutually constituted [19] [introduction chapter]. For Foucault and Massey, space is not articulated as static but as a dynamic and continuous process wherein power and resistance constantly rise and subside just as waves in the ocean do. It is in this constant ebb and flow that meaning is produced, the reason for which it becomes impossible to separate space, meaning, and subjectivity. As Massey points out, space is co-constitutive in the process of identity formation [13]. This article focuses on spaces that challenge hegemonic structures, the reason for which I now turn to Foucault and his idea of heterotopia.

\subsection{Heterotopia, a Place for Resistance}

Foucault formulated ideas on place and space as an arena for resisting hegemony-a kind of counter-space. Such spaces are not in opposition to mainstream society, rather they strive for an extension of traditional dualism: 'the real and imagined merge and give rise to the new, yet un-thought' [20] (p. 14). Foucault [15] talks about these spaces as heterotopias. Heterotopia was originally a medical term that Foucault introduced to the humanities. It translates literally to 'another other place' or 'place of otherness' [20]. Foucault [15] articulates heterotopia as a deviating space that functions as a counter-site reflecting the society in which it is embedded. He places it somewhere between mainstream society and a utopia. As opposed to a utopia, a heterotopia could be found in an actual location, 'outside of all places' [15] (p. 24) and is 'absolutely different from all the sites that they reflect and speak about' [ibid]. Foucault explains a heterotopia as having the quality of being 'in relation with all other sites, but in such a way as to suspect, neutralize or invert the set of relations that they happen to designate, mirror, or reflect' [15] (p.24). He establishes principles of that which constitutes a heterotopia, although he is careful to mention that there are no systematic descriptions of a heterotopia; its function differs between different societies and shifts over time as societies change. An example of such a principle is the idea of a heterotopia as a place of deviation, where people who deviate from the rest of society [such as criminals or the mentally ill] are put into prisons or care homes. Another quality of a heterotopia is that it is 'capable of juxtaposing in a single place several spaces' [15] (p. 25).

The idea of heterotopia could be read in several ways; however, for this article I shall focus on the idea of resistance. Both Kevin Hetherington [21] and Edward Soja [20] articulate the heterotopia as a site of 'otherness' or of 'difference' when compared to the mainstream capitalist society in which it is embedded. By being a site of 'otherness', it has the potential to be a site for transgression, cultural resistance, and alternative identity formations. That is to say, the 'other' is understood as something that is incongruous. The other puts forward something different that challenges conventions. For Soja, a heterotopia is a response to a postmodern world marked by multiple ideological crises. Soja uses the term 'third space' when describing a space that enables emancipatory and radical modes of thoughts and actions other than those in the neoliberal world. The 'other' should not be understood as the opposite of that which already exists but rather as 'drawing on two opposing categories to open up new alternatives' [20] (p. 5). Thus, the heterotopia is here perceived as a site for a collective and critical examination of society as well as a critical resistance-not by an individual but by a group of people.

Central to Hetherington's comprehension of a heterotopia is the idea that it is a site for an ordering that is distinct from that of mainstream society. In line with Foucault, Hetherington puts forth the idea that there is no place that is free of social ordering. A heterotopia is thus not a place of total freedom; rather, it is a site balancing freedom and control, where transformation and resistance are possible. A heterotopia generates an alternative ordering of power, knowledge, and relationships by functioning with its own set of rules, codes, and symbols.

By stating that a heterotopia has its own set of rules and way of ordering things, Hetherington's reading is distinguished from the idea of a utopia, which could be considered to be an unreal site with no ordering and total freedom [comp. More, 1516]. However, a heterotopia may be a site for 
utopic ideas, serving as inspiration when new orderings and identities are being negotiated. For Soja, a heterotopia is a space in which the imagined and experienced merge (which could be part of utopian thinking).

All of these ideas are interesting when researching a drama practice with emancipatory ambitions. By mapping the drama room as a heterotopia, I want to explore the manner in which the drama room could be more than a safe space [comp. Hunter, 2008], where participants, with the leadership of a professional drama teacher, try new roles and challenge themselves by becoming 'the other'. It has the potential to be a space of resistance and, situated between utopia and everyday life, it could also be a dynamic place for knowledge production. By putting space and place in the foreground, my aim is to show how the girls spatialise resistance by letting their everyday lives become the centre of the drama space. The drama space can be understood as a heterotopia for marginalised bodies, experiences, and knowledge. Moreover, putting the marginalised in the centre creates a potential site for other discourse and utopian visions. When exploring the relations between drama, space, and knowledge, in the next section, I map how they coproduce common and individual trajectories among the girls, while also articulating the different spaces that come into being.

\subsection{Mapping the Drama Room}

To explore the relations between drama, space, and knowledge I put cartography to work. Cartography is a method inspired by Deleuze which has been adapted by scholars such as Rosie Braidotti, Hillevi Lenz Taguchi, and Lena Aronsson. For Braidotti [16,17] cartography is a way of mapping what kind of knowing subjects that are produced as they become embedded in a particular context and place-in this case, the girls' drama room. As Nicholson [22] indicates, the field of applied theatre rests on the intersections of culture, community, and identity. Mapping these different aspects is a way of making them explicit and exploring ways in which theory relates to practice. Another layer to my cartography is my investigation of how the drama room can be conceptualised as a heterotopia, that is, as a way of comprehending the multiple ways in which the drama room is either entangled with or isolated from other places in the girls' lives. I do not claim that this cartography is exhaustive. Rather, I explore what subject is in the process of becoming, as well as what is ceasing to be, which can be understood to happen simultaneously in a nonlinear time continuum [17]. This cartography is inspired by Aronsson and Lenz Taguchi [23] who have mapped the encounter between neuroscientific research and early childhood education.

For this article, I interviewed nine girls from the community theatre group. This was done during the spring of 2019 at a local café (This study has been approved by the Swedish ethical vetting committee [Etikprövningsnämnden]). I consider the production of knowledge to be a joint effort in which many lines of thought and embodied practices are woven together by those involved in the research. The girls were not familiar with putting their drama practice into words; hence, they sometimes found it difficult to formulate their thoughts [as Hedda sighed, 'It's hard to put everything into words, the words don't cover all the things we do']. When this happened, I would, together with the girls, try to find suitable words. Sometimes we explored the questions together. To be certain that I had understood them correctly, I would summarise their comments in my own words and then allow them the opportunity to agree or disagree. That way, I was part of the construction-not only of the empirical material but also of the girls' recollection of their theatre practice in the drama space. As Braidotti [16] makes clear, memories are always constructed with others, and my role as a researcher is neither neutral nor innocent. I recognise that, as a researcher, I am responsible for analysing the empirical material [films, transcribed interviews, etc.]. It is from my position as a researcher that I choose what threads to analyse from the material that has been constructed. I am the one making the cuts from a larger entanglement 'splitting and unwinding among many threads momentarily separating them' [24] ( $\mathrm{p} .73$, own translation) to better comprehend the becoming of the different spaces of the drama room. Another layer of the construction of the material is the translation of the girls' 
statements as the interviews were conducted in Swedish. I have tried my best to capture not only what they have said but also how they speak about and reflect on their drama space.

\section{Results}

\subsection{Articulating the Drama Room As a Heterotopia}

As became clear when talking with the girls, the drama room fills many functions, allowing multiple trajectories to coexist. The cartography points at how the drama space is in a constant becoming in relation to other places of significance in the girls' life, allowing a particular knowledge to emerge. After listening to the girls, I tried to sort their answers into different themes. Three heterotopic functions of the drama space materialised: an exclusive space for girls only; a space for multiple, affective, and embodied stories; and, a space for resistance.

My themes have shifted during this process as I have encountered the girls' stories. The chosen quotations illustrate a larger reoccurring picture. All themes are related, with one being unable to exist without another. However, for the sake of clarity, I untangle the threads and explain them separately.

\subsection{The Exclusive and Including Girl-friendly Space}

When interviewed, the girls talk about the drama space with enthusiasm. Being in the drama room is connected to feelings such as joy, creativity, and participation. Although drama and theatre practice is important, other values are brought to the fore as well. When trying to articulate the drama space, many of the girls use words such as 'home', 'family', 'friendship', and 'freedom to be myself'. A sense of community and friendship permeate the interviews. Sarah says that the girls in the studio are the younger sisters she never had. Nora shares her experience of openness in the drama room:

The girls and the space we are in. It is just for us and ... there is no one there but us and there are no other people running around because it's excluded... It's just that space. It's a peaceful place. You leave everything else outside that room.

With the room being just for them, and with the fact that they are all girls, they are provided with a sense of 'exclusive inclusion'. The drama room is situated so that they are not interrupted by others and therefore creates a sense of leaving the rest of the world outside. In this respect, the drama space bears similarities to the idea of a safe space, as put forth by Hunter [25]. This isolation is, as Hunter indicates, vital for creating a sense of safety and 'us' as well as allowing messy negotiations to take place. When talking about the drama space in this positive manner, there is a more or less explicit comparison with their school. As Lea explains:

I can be myself there and no one is judging me, at the theatre everybody can relate to each other. In school, there is bullshit, and there are so many other students there why I'm always on the watch-out.

The production of the drama room as being a non-judgemental space permeates the interviews. The interviewed girls attend the drama workshops regularly, and they are able to forge a sense of intimacy with the other members of the group, which does not seem possible at school. This way, the drama room positively reaches across as a highly relational space. What also contributes to a sense of 'family' and 'friendship' is the girls feeling comfortable in the drama space, which enables a sense of relaxation. While Lea is general in her comparison, Alya is more specific when identifying her appreciation of the drama room as a positive relational space:

In school, it's like the girls and boys are all mixed but the boys will take up most of the space. And among the girls, it is a bit complicated... At the theatre, we are all allowed to take our place even though one is not very loud or extroverted. People will listen to you and take it seriously.

Alya's statement can be understood as a reaction against boys occupying most of the space in school. However, there is also a reaction involving girls. The drama room is perhaps not only a space 
for the girls to escape the male gaze but also to escape schemes between other girls. It seems that the drama room is a space for being and becoming in another way, beyond judgement and false-play. Being allowed to take their place, and being listened to by the leaders and each other, creates a space where the girls can set the agenda on their own terms. This space facilitates the creation of the 'other social ordering' that is vital for a heterotopia [21]. The 'oppressive other' is considered in this process of disordering and decentring, thereby allowing the girls to construct a different sense of subjectivity $[20,26]$ which could be important in their everyday life. The girls' voices are being moved from the margins to the very centre of attention, which is crucial for creating a sense of freedom, agency, and relaxation. This deep sense of relaxation, freedom, and feeling 'at home' seems to be connected to this particular drama space in the girls' lives. I believe that the girls' feeling that the drama room is their place, allowing them to shape the process in their own way, is one aspect that enables the aforementioned sense of relief and enjoyment to set in. By being a separate place, a different space can be produced that is a counter-site, inverting and juxtaposing the social orders permeating the girls' everyday places. This is not a utopian space; it has its own set of regulations and ways of ordering that need scrutiny. However, the ideas emerging from this space could be utopic, which are explored later in the article.

\subsection{A Space for Becoming through Multiple Embodied Stories}

While the drama space has qualities of being a safe and isolated space and being in opposition with other spaces, the following functions, when mapped out, show an explicit and transversal entanglement with other places in the girls' lives. Central here is the expression and embodiment of emotions in the drama room. Many of the girls come into the drama room with emotions and experiences from different areas of their lives although school is central. These experiences and feelings are processed and cultivated when participating in drama activities, which offer a sense of relief and purification that is in line with a heterotopia. This could also be linked to the idea of perezhivanie, which is a term from pedagogue Lev Vygotsky and theatre director and pedagogue Constantin Stanislavsky that is sometimes applied to drama [27]. The term translates as 'intensely-emotional-lived-through-experience' [Ferholt cited in [27] (p. 10) which can be understood to be an experience not easily forgotten. Therefore, it could be both purifying and transformative. For Stanislavsky, this affective experience is a tool for the actor to use on stage when they embody a character [27]. For Nora, the frustration that has built up during the school week and experiences in her spare time serves as sources for inspiration in the drama room as the room became a 'place to let it all out in the shape of creativity'. When embodying feelings and experiences from other places in an improvisational exercise, Nora experiences a sense of genuineness, which, in turn, is a 'stress-relief', where she can just let go of it all. This physical affective aspect is also of importance to Sarah:

I can show myself beyond words at the theatre; physically with my body and with emotions' ( ... ) 'It just feels like such a relief to be there' (...) 'I can take my anger and mediate it to something that can be understood by others. I have also learnt to express myself and my emotions with my body.

What is in the making is a space in which expression through bodies and emotions matters. It is not only a space 'to let it all out', as Nora explained, but also a space to embody the girls' experiences and create new expressions as in Sarah's case. The physicality of their expressions allows the girls to express themselves beyond words, create and recreate experiences, and create new stories that can be put in motion [16].

Another aspect that is being brought forth is the everyday life being carried out at school and in the town where the girls live. Many of them explain the happenings and interactions that they observe on the bus or in the school corridor as 'normal' or 'regular'. When bringing those experiences into the drama room, they can reflect these places. This reflection serves as inspiration to create scenes and characters in the drama room, which, in turn, is a way to deal with everyday life. For Alya, it was a way to create characters like herself and tell her story of the town: 
I am only in this town all the time... it's like ... a lot to tell about this town, what has happened here. What has happened in school with my mates or teachers.

The feelings, reflections, and experiences that the girls materialise together are vital as they serve as a kind of raw material that is processed. When putting their stories into motion, and thus creating new stories, a bodily knowledge is able to emerge. The themes emerging from everyday life range from issues such as hair removal in relation to the hair norms among the peers in their schools to virginity controls and sexuality connected to the culture in their town. Matters that are explored in the drama room are reworked into shorter scenes and often they become part of the public performance. Learning to master one's voice and embody emotions when working on a scene is a way of refining the emotions and experiences that may not initially be comprehensible to the girls. The space in the drama room can thus be understood as a space that comes into being by purifying and reordering disordered experiences and emotions, mainly from school but also from other spaces in their town, their home and their spare-time activities. During this reordering procedure, which could be conceptualised as a perezhivanie, a refining of emotional expression occurs. Refining is here considered to be a way of producing skills and knowledge that are not fixed but constantly evolving from the particular context of heterotopia. I call this 'dramaspaceknowledge': a knowledge emerging in a particular drama space. Refining in the drama room happens as emotions and experiences become tools to embody and create a theatrical narrative. Being able to embody and voice experiences can be considered to be 'dramaspaceknowledge' and is crucial when shaping those experiences into a scene that is to be in the play or an improvisational exercise. Linn explains:

We are creating a performance out of our own experiences, from what we've heard, seen and our thoughts and then we like go into different characters and like ... . Create a performance.

There are many threads involved in the creation of a performance, which is not limited to the girls' experience. In addition, their peers who are not present in the room seem to be given voices. Thoughts and experiences become embodied, shaping not only new stories but also new characters to inhabit them. The drama space becomes a space that holds many embodied stories, thus capturing multiple truths. As this is a continuous process, the drama space is constantly in a state of becoming [13]. By engaging in this space, the girls might enact multiple identities as they explore different characters and stories. The girls' ability to hold multiple truths is linked to empathy, which I consider to be a 'dramaspaceknowledge'. I attend to this in the following section.

It is in this iterative and artistic space, constantly in the making, that many of the girls are able to express knowledge that will be embodied when they develop and perform a play. This can be understood as part of a transforming process, which is important for alternative subject formation [21]. Creating characters, the girls experiment with a 'dual effect' where they are in character and within themselves at the same time [28]. By developing the skills needed to inhabit a role, the girls become knowing subjects $[16,17]$ who are capable of embodying their own and others' experiences. Having a sense of control over the emotional body, as Sarah expressed, is intertwined with the sense of freedom that many of the girls experience in the drama room when they use their bodies freely while improvising. Freedom and control, as Hetherington points out, are vital in a heterotopia for any kind of transformation to occur. Rather than exploring freedom and control as binaries constituting each other, I perceive them as being vital for creating the 'other', a third option of the not-yet-thought, which, in this case, materialises in embodied, improvised scenes. This continuous interplay between freedom and control is important when producing dramaspaceknowledge.

Dramaspaceknowledge empowers the girls to enact and voice their feelings beyond the drama room or the stage. Dramaspaceknowledge is directly applicable to school. When doing a presentation, Hedda takes advantage of both her voice skills and her ability to act in role:

It feels a little bit easier if I think of it as if I'm acting and then it's not me who is doing the presentation and it's like I can't do anything wrong. 
Hedda controls her body and voice while also articulating a knowledge of role-creation, which protects her from a situation that she might experience as threatening by making herself at home in a role where she cannot do anything wrong because roles are fictional. This can be seen as a way for her to navigate the school system where there are clear boundaries between what is right or wrong and between failure or success.

As a space for multiple stories and truths, the drama room offers an important possibility for embodying a diversity of narratives and characters. These stories reflect and juxtapose various other sites, in line with Foucault's idea of a heterotopia. Although mapped as a separate space in the previous section, it is clear that the drama space cannot exist on its own but must be entangled with other sites to be a dynamic space of movement and stories. Emotions, experiences, and interactions at school in particular but also in the town are disentangled in the drama space and returned to school in the shape of skills. This is particularly obvious when it comes to doing presentations and in some cases when writing. I do not analyse this entanglement as a circular movement. Instead, the lines work in transversal ways, going back and forth, constantly reshaping themselves. It is in this constant flow that the knowing subject emerges, nomadic in her nature as she engages with an entanglement of multiple spaces and places.

\subsection{A Political Space for Resistance and Dialogue}

A trajectory of emancipatory and critical thinking stretches across all of the interviews with the girls. When embodying their everyday life each week in the drama room, reproductions of inequity appear, from which critical emancipatory thinking emerges. Many of the girls are rupturing the limiting structures that they find themselves entangled with as girls. Hedda says that being part of the drama group has helped her understand 'about other cultures and other backgrounds and their conditions and what that is like. I am a girl ... and I have come to understand how that will affect me-what it means to be a girl when other puts you in a structure'. Hedda articulates a knowledge regarding other cultures and the reason for which people behave in different ways. This knowledge was also expressed by many of the other girls in the group. She also comprehends that there are structures affecting her as a girl. These findings are crucial for the performance that the girls are in the process of creating, and this kind of dramaspaceknowledge comes to permeate their everyday lives in various ways. Learning about each other's differences has been important in the creation of empathy and in the cultivation of a non-judgemental attitude towards their peers in school. For some, this is a tool for conflict management. Being able to see through structures has also helped the girls stand up for themselves, as Sarah explains:

If somebody in school touches me I can say no to that person. I wouldn't have dared to do that if it hadn't been for the theatre because then I would have thought that they wouldn't notice my objection and just continue. Now I can speak up for myself and mean it.

Sarah's statement demonstrates the emergence of an emancipated voice. She knows how to speak up for herself in a confident way. She is able to enact resistance to protect herself, thus challenging the peer culture at school. Whereas Hedda expresses how she uses the dramaspaceknowledge to manage school tasks, Sarah handles the social aspect of school by standing up for herself. The girls become knowing subjects in different ways. Alya is one of the girls who takes this one step further by not only speaking up for herself but also challenging the system. By embodying her and others' experiences in the drama space, she has become aware of sexist and oppressive structures at school. This has led to confrontations with teachers. She explains that sometimes the teachers listen and act on what she sees, but at other times she feels ignored:

They don't take me seriously, because I'm like ... just a child, a pupil, and they are adults and they are like 'you are small and don't understand' and that is kind of hard.

Alya's experience of speaking up against the system and not being heard shows a tension between the 'freedom' experienced in the drama space and a school space with a different hegemonic ordering. 
When talking to Alya about this tension, there seems to be an ostensible reconciliation, and she explains that she keeps on challenging the system despite not always being heard.

In different ways, the girls are expressing a resistance to a hegemonic system that, from their perspective, does not work in their favour. By bringing experiences from their everyday places to the drama room, the drama room becomes 'capable of juxtaposing in a single real place several spaces' [15] (p. 25). By embodying and enacting experiences from their everyday lives, limiting structures are laid bare for scrutiny. With the ability to talk about these structures in a safe and separate space, the girls are now breaking the glass ceiling of foisted identity to imagine a new way of ordering. I believe this emancipatory and critical thinking adds another layer to the dramaspaceknowledge. By enacting this other way of being in the drama room, the girls put this different ordering into practice, thereby creating space for the production of emancipation and agency. Again, I would not call this a utopic space as new social ordering takes place. However, the thinking that is happening may well be utopic. Creating utopic visions may, in turn, create powerful juxtapositions between what is and what could be, thus inspiring the girls' agency to extend to their everyday life. Articulating the drama room as a space for utopic thinking turns it into a powerful counter site to the other visions presented by the neoliberal thinking that permeates society [29]. Again, this does not mean that the drama space is a utopia! The drama space can be understood as coproducing new voices and actions and creating knowing as well as visionary subjects. Just as with the embodied knowledge mentioned above, the nomadic subject transfers this knowledge to other sites. When translated to other sites, the knowledge becomes a protective shield or part of a dialogue to avoid conflict. For some, such as Alya, the dramaspaceknowledge becomes opposition against a system-and this is not easy. Perhaps this is because the girls are acting as individuals in their different schools, which is not enough to change structures and normative representation.

\section{Closing Reflections}

In this paper, I have mapped the drama room belonging to an all-girl community theatre group as a heterotopia. Putting space and place in the foreground makes it possible to explore ways in which knowledge works in a transgressive way between the drama space and the everyday life of the girls. The drama space is constructed by being closely linked to those spaces of everyday life although school stands out. School and the drama room are deeply entangled, and by interacting in these different settings, the girls become not only knowing subjects but also visionary subjects. Visionary subjects know that a space with a different social ordering is possible and are encouraged to act and see through oppressive systems. In addition, they are able to embody a dramaspaceknowledge by using their voices and bodies to manage school. Though the girls operate differently, they have a wide range of trajectories available to them. However, this would not have been possible without the drama room. The drama room is at once both deeply ensconced with and isolated from other sites. As exploring the drama room has made clear, this contradictive relation is vital for a heterotopia.

As a 'dramapractionerresearcher', I wonder whether we as practitioners are aware of the entanglement of the drama room with other places of significance in our participants' lives? What ethical implication does this have for our practice? If we create spaces that produce knowing and visionary subjects, we must also know what other places these subjects embody and ensure that they, as nomadic subjects, move swiftly between the different social orderings in different contexts. Soja and Hetherington encounter heterotopia as the 'other space' in relation to mainstream capitalistic and neoliberal society; however, I would argue that this is too vague. We have to be more precise when articulating the specific places that the drama room is entangled with or isolated from. Do we know what places the drama space reflects, juxtaposes, or deviates from? This raises questions regarding the extent to which dramaspaceknowledge can stretch and the farthest that our responsibilities as drama practitioners can reach. I do not have all of the answers, but encountering the drama room as a heterotopia urges us to consider these matters. 
Funding: This research received no external funding.

Acknowledgments: The author wishes to thank ass. Senior lecturer Karin Gunnarsson and Senior Lecturer Ulrika von Schantz at Stockholm University, Sweden, for their kind and professional support when writing this article. Many thanks also goes to Professor Helen Nicholson at the Royal Holloway University of London, UK, for her encouragement during the writing process.

Conflicts of Interest: The author declares no conflict of interest.

\section{References}

1. Neelands, J. Acting together: Ensemble as a democratic process in art and life. Res. Drama Educ. J. Appl. Theatr. Perform. 2009, 14, 173-189. [CrossRef]

2. Neelands, J.; O'Connor, P. Creating democratic citizenship through drama education: The writings of Jonothan Neelands. In Stoke on Trent: Trentham Books; Trentham Books Ltd.: London, UK, 2010.

3. Way, B. Development through Drama; Humanity Books: Amherst, NY, USA, 1998.

4. Burton, B. Dramatising the hidden hurt: Acting against covert bullying by adolescent girls. Res. Drama Educ. J. Appl. Theatr. Perform. 2010, 15, 255-270. [CrossRef]

5. Hatton, C. Performing 'Girl' in the Facebook Era: Drama as a Safe Space for Negotiating Adolescent Identities and Agency. NJ Drama Aust. J. 2012, 36, 36-49. [CrossRef]

6. Hatton, C. Educating Rita and her sisters: Using drama to reimagine femininities in schools. Res. Drama Educ. J. Appl. Theatr. Perform. 2013, 18, 155-167. [CrossRef]

7. Gallagher, K. Drama Education in the Lives of Girls; University of Toronto Press Inc.: Toronto, ON, Canada, 2001.

8. Hatton, C. Can I get a witness? Mapping learning in and beyond the drama classroom. J. Artist. Creat. Educ. 2007, 1, 34.

9. Grünbaum, A. Lika och unika: Dramapedagogik om Minoriteter; Daidalos: Göteborg, Sweden, 2009.

10. Sternudd, M.M.F. Dramapedagogik som demokratisk fostran? fyra dramapedagogiska perspektiv: dramapedagogik i fyra läroplaner. [Educational Drama as a means of fostering democraticvalues? Four perspectives in educational drama—educational drama in four curricula]. Ph.D. Thesis, Acta Universitatis Upsaliensis, Uppsala, Sweden, 2000.

11. Cohen-Cruz, J. Local Acts: Community-Based Performance in the United States; Rutgers University Press: New Brunswick, NJ, USA, 2005.

12. Read, A. Theatre and Everyday Life; Routledge: London, UK, 1993.

13. Massey, D.B. For Space; SAGE: London, UK, 2005.

14. Eriksson, C. A Preschool that Brings Children into Public Spaces: Onto-Epistemological Research Methods of Vocal Strolls, Metaphors, Mappings and Preschool Displacements. Ph.D. Thesis, Stockholm University, Stockholm, Sweden, 2020.

15. Foucault, M.; Miskowiec, J. Of Other Spaces. Diacritics 1986, 16, 22. [CrossRef]

16. Braidotti, R. The Posthuman; Polity: Cambridge, UK, 2013.

17. Braidotti, R. A Theoretical Framework for the Critical Posthumanities. Theory Cult. Soc. 2018, 36, 31-61.

18. Deleuze, G.; Guattari, F. Kapitalism Och Schizofreni Tusen Platåer; Tankekraft: Hägersten, Sweden, 2015.

19. Hinton, P.; Treusch, P. (Eds.) Teaching with feminist materialisms: Teaching with gender. In European Women's Studies in International and Interdisciplinary Classrooms; ATGENDER: Utrecht, The Netherlands, 2015.

20. Soja, E.W. Thirdspace: Journeys to Los Angeles and Other Real-and-Imagined Places; Blackwell: Cambridge, MA, USA, 1996.

21. Hetherington, K. The Badlands of Modernity Heterotopia and Social Ordering; Routledge: London, UK, 1997.

22. Nicholson, H. Applied Drama: The Gift of Theatre; Palgrave Macmillan: Basingstoke, UK, 2014.

23. Aronsson, L.; Taguchi, H.L. Mapping a collaborative cartography of the encounters between the neurosciences and early childhood education practices. Discourse Stud. Cult. Politi Educ. 2017, 39, 242-257. [CrossRef]

24. Gunnarsson, K. Med önskan om kontroll: figurationer av hälsa i skolors hälsofrämjande arbete [With Desire for Control, Figurations of Health in School Health Promotion]. Ph.D. Thesis, Stockholms Universitet, Stockholm, Sweden, 2015.

25. Hunter, M.A. Cultivating the art of safe space. Res. Drama Educ. J. Appl. Theatr. Perform. 2008, 13, 5-21. [CrossRef]

26. Bell, H. Yearning: Race, Gender, and Cultural Politics; South End Press: Boston, MA, USA, 1990. 
27. Hallgren, E. Ledtrådar till estetiskt engagemang i processdrama: samspel i roll i en fiktiv verksamhet. [Clues to Aesthetic Engagement in Processdrama, Joint Action in Fictional Activity]. Ph.D. Thesis, Stockholms Universitet, Stockholm, Sweden, 2018.

28. Bolton, G.M. Drama as Education an Argument for Placing Drama at the Centre of the Curriculum; Longman: Harlow, UK, 1984.

29. Hancock, D.; Faramelli, A.; White, R. Spaces of Crisis and Critique: Heterotopias beyond Foucault; Bloomsbury Academic: London, UK, 2018.

(c)

(C) 2020 by the author. Licensee MDPI, Basel, Switzerland. This article is an open access article distributed under the terms and conditions of the Creative Commons Attribution (CC BY) license (http://creativecommons.org/licenses/by/4.0/). 